\title{
Interactive comment on "Evaluation of a field-deployable Nafion-based air drying system for collecting whole air samples and its application to stable isotope measurements of $\mathrm{CO}_{2}$ " by Dipayan Paul et al.
}

Dipayan Paul et al.

d.paul@rug.nl

Received and published: 20 May 2020

The comment was uploaded in the form of a supplement:

https://www.atmos-meas-tech-discuss.net/amt-2019-295/amt-2019-295-AC2-

supplement.pdf 